\title{
The Impacts of Burst Assembly Parameters on Optical Burst Switching Network Performance
}

\author{
Mohammed Al-Shargabi \\ Department Of Information Systems, \\ College of Computer Science and Information Systems, Najran University \\ mashargabi@nu.edu.sa
}

\begin{abstract}
An Optical Burst Switching (OBS) network is one of the newer generations of optical networking paradigm, which is a new type of optical internet infrastructure. A burst assembly is a process occurring inside an OBS network, which gathers the packets to create the data bursts. The ingress node conducts the burst assembly by sequencing the packets arriving at the different destination queues. Based on several methods, the ingress node sends the data burst as one unit to the core nodes. Therefore, the core nodes receive fewer requests. Although, the OBS burst assembly parameters result in traffic characteristics (e.g., burst size) which impacts on network performance. Consequently, this paper surveys OBS networks burst assembly techniques in detail. Moreover, an investigation study examining the effect of burst size on reducing the OBS network packet's loss probability is an important element affecting OBS performance which will be introduced. The study has been simulated with a number of traffic load rates, which are: incremental load, high load, average load, and low load to ensure the accuracy. The investigation results demonstrate that small burst size performs better than large burst size.
\end{abstract}

Key words: Optical Burst Switching (OBS) Network; Burst Assembly; Burst Size; Network Performance

\section{INTRODUCTION}

An OBS network is a new generation of optical networking paradigm[1] combining the benefits of Optical Circuit Switching (OCS) and Optical Packet Switching (OPS) methods. Its aims are to better utilize wavelengths, minimizing latency (setup delay), and avoiding the use of optical buffers. OBS is also designed to combine various traffic types in the same burst and to isolate QoS for each traffic type. Reducing network packet loss probability in an OBS network is a crucial element for guaranteeing the QoS for any type of internet traffic. Several signalling schemes have been proposed to allocate recourses to avoid the contention of the traffic [2-6]. However, if multiple bursts from different input ports are simultaneously sent to the same output port, an issue known as 'contention' (blocking) will arise. Thus, several methods are suggested to conduct the burst assembly process to reduce the probability of packet loss, as such time-based schemes [7], threshold-based schemes [8], hybrid time and threshold-based schemes [9], learning-based burst assembly (LBA) [10], and averagedelay-based burst assembly schemes [11]. However, these studies do not investigate the impact of the burst size on OBS network performance. Thus, a detailed survey on OBS networks burst assembly techniques is provided as well as an investigation into the effect of burst size on reducing the OBS network packet loss probability as the primary factor affecting OBS performance.

This paper is divided into the following sections: section 2 details literature on OBS assembly schemes. The simulation model and the results and discussion are in section 3. Finally, section 4 presents the conclusion.

\section{OBS ASSEMBLY SCHEMES}

The time-based scheme [7] uses an interval time, $\mathrm{T}$ for creating the data burst. Every packet which arrives in the interval time T, will be used for creating the burst. Each of the destination queues has its own timer which is initiated from 0 . During the interval time, every packet with the same destination will queue respective of its destination. When the timer arrives at $\mathrm{T}$, the queued packets are incorporated into the data burst. This strategy has a long burst length if the input traffic is high since it increases the loss rate in the core node. As well as this drawback, the small burst size in the low input traffic creates a large amount of control packets, increasing the process in the core node.

The threshold-based scheme [8] employs the Burst minimum size, Bmin parameter, which represents the minimum number of bytes for creating the data burst. Every packet is sequenced in the destination queue until the number of bytes achieves the Bmin parameter value; imposing a delay time is the main disadvantage of this strategy. Hybrid time and threshold/based strategies are suggested [9] to reduce the limitations of the two earlier strategies.

In the hybrid time and threshold-based scheme [9], the burst is created when the timer achieves the maximum value of time Tout or when the amount of bytes achieves the maximum value of Bmin. If the timer achieves the maximum value of $\mathrm{T}$ in the first instance, then it compares the amount of bytes with Bmin. However, if the number of 
bytes is greater than Bmin value, every packet in the destination queue is gathered to create the data burst. Otherwise, creating the data burst will be delayed to the next interval time.

A learning-based burst assembly (LBA) [10] proposed an algorithm to adapt the burst assembly time based on the network's observed loss pattern. It employs an algorithm model which uses learning automata to periodically examine loss in the network and change the assembly time at the ingress node to a favourable one. Choosing an assembly time parameter value is updated dependent upon loss measured over the path using a linear reward-penalty. The benefit of this assembly scheme is that it reduces burst loss probability in comparison to the other adaptive assembly mechanism. Conversely, this scheme does not consider any of the traffic delay requirements. Therefore, the LBA scheme is unable to be used for real time traffic.

The average-delay-based burst assembly scheme [11] proposed burst assembly algorithm on the basis of the average delay of the packets which comprise a burst. The algorithm calculates the Tout based on a variable called TAVE, which may vary for each of the interval times. TAVA can be calculated as follows [12]:

$$
\text { TAVA }=\frac{\text { T'out }}{2}+\frac{1-\mathrm{e}^{-\lambda \text { Tout }}}{2 . \lambda}
$$

where, $\lambda$ is the value of the arrival traffic rate in a specific interval time. The proposed algorithm results in an improvement to the delay jitter of the packets during the burst assembly procedure, in comparison to that of timerbased and burst length-based assembly policies in the TCP traffic. The drawback of this algorithm is its potential suitability for TCP traffic, which estimates the round-trip times. However, it is unsuitable for real time traffic which uses user datagram protocol (UDP) and avoids the roundtrip times. Here, this scheme is not used for the comparison, as it calculates the Tout value based on the round-trip times which is unsuitable for real time traffic.

Researchers in [13] studied the burst length estimation analysis, whilst over-reservation and average wait time are kept to a minimum using asymptotic analysis, whereby the burst length is fixed. However, It did not indicate how to choose the value of the burst size.

In general, the OBS burst assembly parameters result in having control over the traffic characteristics (for instance, burst interval time and the burst length) which impact upon network performance. None of these indicated schemes indicates how to select parameter value (especially burst size). Therefore, an investigation should be conducted to research the impact of burst assembly parameters on OBS network performance.

\section{SIMULATION MODEL AND RESULTS}

In this part, a simulation model using an NCTUns simulator is used for evaluating the impact of burst size on reducing OBS network packet loss probability, as an important factor affecting OBS performance. The OBS network configuration simulation parameters are detailed in Table I.
Table 1 : OBS network configuration simulation parameters

\begin{tabular}{|l|l|}
\hline Parameter & Value \\
\hline Link bandwidth & $1000 \mathrm{Mb} / \mathrm{s}$ \\
\hline Propagation delay & $1 \mu \mathrm{s}$ \\
\hline Bit error rate & 0 \\
\hline Maximum burst length & $\begin{array}{l}4000 \text { Kbyte }-16000 \\
\text { Kbyte }-32000 \text { Kbyte }\end{array}$ \\
\hline Use of Fiber Delay Line (FDL) & No \\
\hline Use of Wavelength Conversion & No \\
\hline
\end{tabular}

In the traffic configuration, trace files are created with several traffic load rate, thus: incremental load, high load (simulator maximum bandwidth), average load, and low load. The impact of burst size has been studied under all viable traffic load rates as stated above to ensure result accuracy within any traffic load.

The results show that the probability of packet loss is less when a small burst size is used. Figures 1 and 2 show a comparison of packet loss probability among the different sizes using incremental load rate. The results of the packet loss probability when the burst size is $4000 \mathrm{~Kb}$ is lower than other burst sizes in all of the traffic loads.

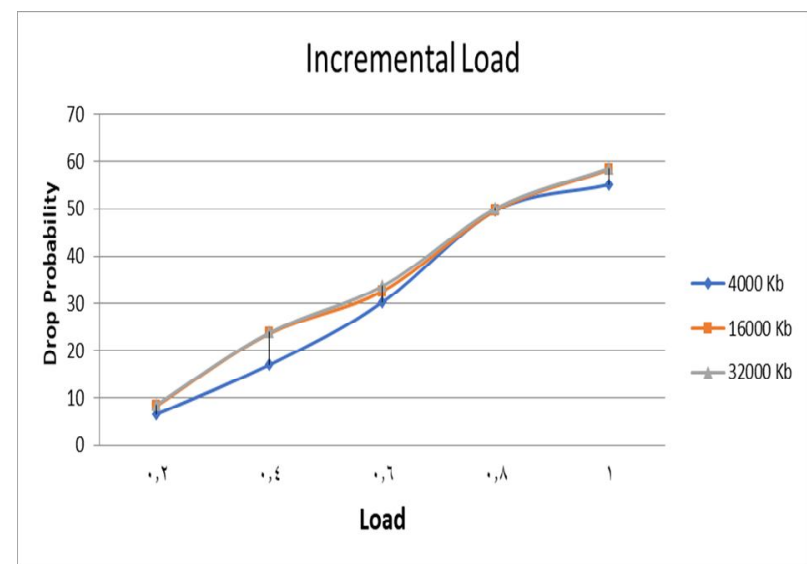

Figure 1 :The packet's loss probability using several burst sizes using incremental load rate

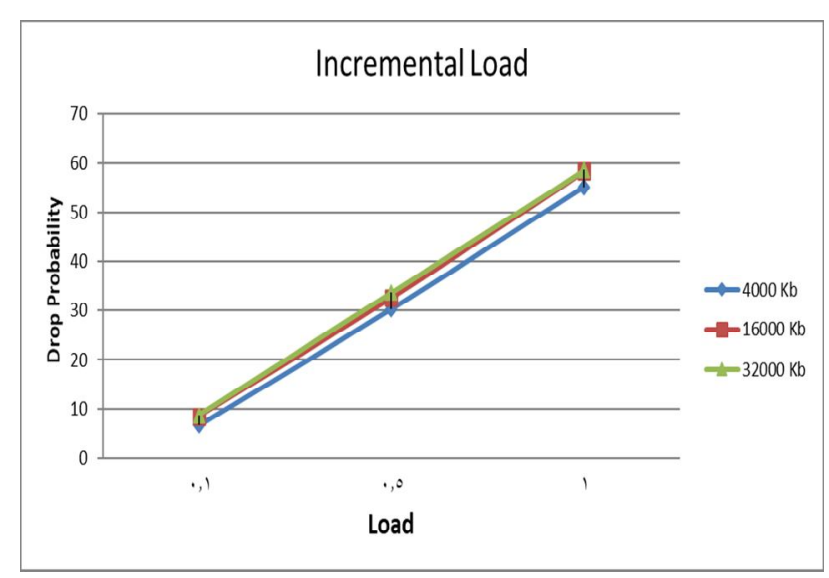

Figure 2: The packet's loss probability using several burst sizes using incremental load rate (different view) 
Additionally, a simulation model is used to evaluate the impact of burst size on reducing the loss probability of OBS network packets under high load, average load, and low load. Figures 3 (low load), 4 (average load), and 5 (high load) also indicate that the packet loss probability results when the burst size is $4000 \mathrm{~Kb}$ is lower than the other burst size in all of the traffic loads.

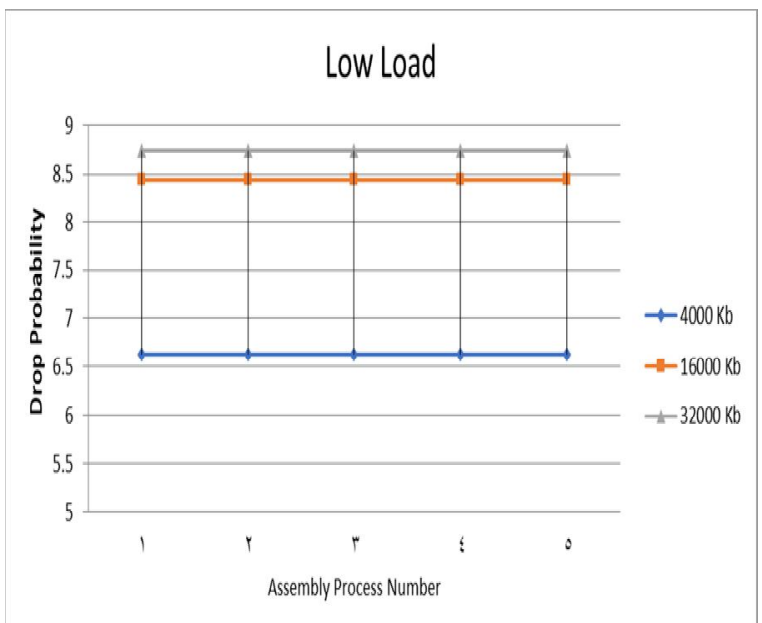

Figure 3 :The packet's loss probability using several burst sizes using low load rate

The results demonstrate that network performance in regard to packet loss probability is improved when a small burst size is used. This is because a small burst size consumes fewer resources time in the obs core nodes in comparison to medium or large burst size which consumes more resources time in the obs core nodes leading to more contention (more packet's loss probability) when other bursts from other input ports are simultaneously used for the same output port.

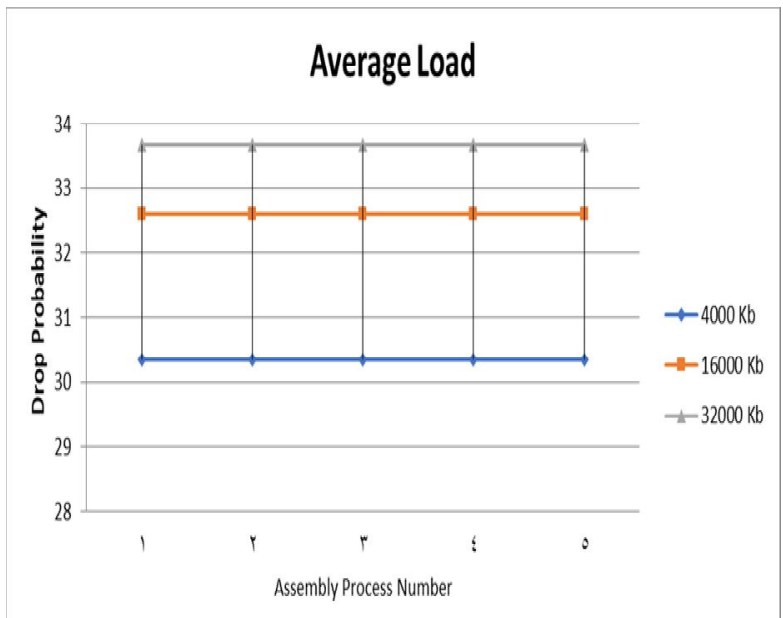

Figure 4:The packet's loss probability using several burst sizes using average load rate

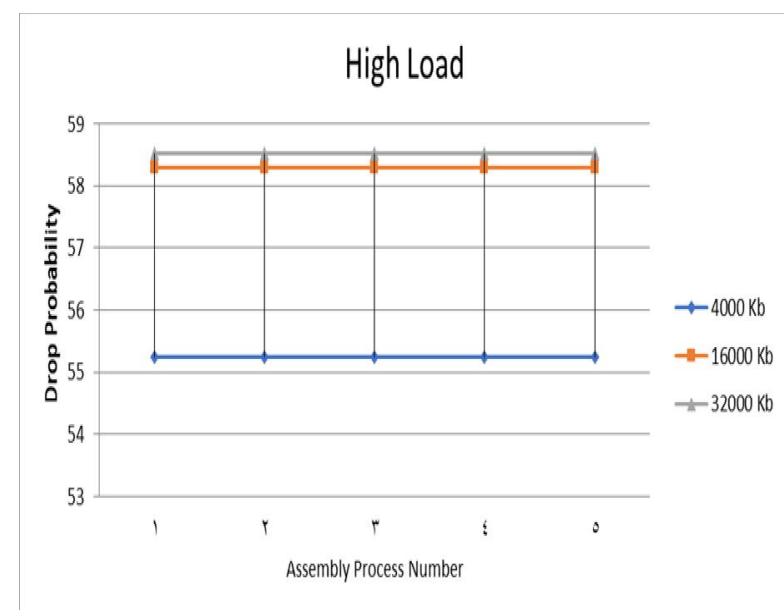

Figure 5: The packet's loss probability using several burst sizes using high load rate

\section{CONCLUSION}

This paper has investigated the effects of burst size on reducing OBS network packet loss probability as a primary element affecting OBS performance. The study was simulated with several traffic load rates: incremental load, high load, average load, and low load to investigate the impact of burst size under all traffic load rates, and to guarantee the accuracy of the results within any traffic load. The investigation results indicate that small burst size can perform better than large burst size because of the small burst size consuming fewer time resources in the OBS core nodes compared to the medium or large burst size which consumes more resources time in the OBS core nodes, which leads to greater contention (more packet loss probability) when other bursts from other input ports are simultaneously sent to the same output port.

\section{REFERENCES}

[1] Qiao, C., and Yoo, M. Optical Burst Switching (OBS) - a new paradigm for an optical Internet. Journal High Speed Networks, vol. 8, 1999. pp. 6984.

[2] WEI, John Y.; MCFARLAND, Ray I. Just-in-time signaling for WDM optical burst switching networks. Journal of lightwave technology, 2000, 18.12: 2019-2037.

[3] DUTTA, Manoj Kr. A Comparative Study Among Different Signaling Schemes of Optical Burst Switching (OBS) Network for Real-Time Multimedia Applications. In: Advances in Computational Intelligence. Springer, Singapore, 2020. p. 107-117.

[4] DUTTA, Manoj Kr. A Comparative Study Among Different Signaling Schemes of Optical Burst Switching (OBS) Network for Real-Time. In: Advances in Computational Intelligence: Proceedings of Second International Conference on Computational Intelligence 2018. Springer, 2019. p. 107.

[5] P. Srikanth Reddy, et al. Study and Analysis of Routing Protocols, International Journal of Emerging Trends in Engineering Research, 2019. Volume 7 No. 11. 
[6] N V V N J Sri Lakshmi, et al. Study and Analysis of Defence Techniques for Various Network Topologies, International Journal of Emerging Trends in Engineering Research, 2019. Volume 7 No. 11.

[7] DUSER, Michael; BAYVEL, Polina. Performance of a dynamically wavelength-routed optical burst switched network. IEEE Photonics Technology Letters, 2002, 14.2: 239-241.

[8] GE, An; CALLEGATI, Franco; TAMIL, Lakshman S. On optical burst switching and selfsimilar traffic. IEEE Communications Letters, 2000, 4.3: 98-100.

[9] KANTARCI, Burak; OKTUG, Sema. Adaptive threshold based burst assembly in OBS networks. In: 2006 Canadian Conference on Electrical and Computer Engineering. IEEE, 2006. p. 1419-1422.

[10] VENKATESH, T.; SUJATHA, T. Lakshmi; MURTHY, C. Siva Ram. A novel burst assembly algorithm for optical burst switched networks based on learning automata. In: International IFIP Conference on Optical Network Design and Modeling. Springer, Berlin, Heidelberg, 2007. p. 368-377.

[11] Christodoulopoulos, Konstantinos, Emmanouel Varvarigos, and Kyriakos Vlachos. "A new burst assembly scheme based on the average packet delay and its performance for TCP traffic." Optical Switching and Networking 4.3-4 (2007): 200-212.

[12]CASONI, Maurizio; RAFFAELLI, Carla. TCP performance over optical burst-switched networks with different access technologies. Journal of Optical Communications and Networking, 2009, 1.1: 103-112.

[13] SHUKLA, Vaibhav, et al. Estimation of Burst Length in Optical Burst Switching Networks Based on Early Release of Control Packet. Journal of Engineering Research, 2018, 6.1. 